\title{
Estimating uncertainty in radiation oncology dose prediction with dropout and bootstrap in U-Net models
}

\author{
Alyssa Vanginderdeuren ${ }^{1}$, Margerie Huet-Dastarac ${ }^{2}$, \\ Ana Maria Barragan-Montero ${ }^{2}$, and John A. Lee ${ }^{1,2} *$ \\ 1- UCLouvain.be - ICTEAM \\ Place du Levant 3, 1348 Louvain-la-Neuve - Belgium \\ 2- UCLouvain.be - IREC/MIRO \\ Avenue Hippocrate 55 B1.54.07, 1200 Brussels - Belgium
}

\begin{abstract}
Deep learning models, such as U-Net, can be used to efficiently predict the optimal dose distribution in radiotherapy treatment planning. In this work, we want to supplement the prediction model with a measurement of its uncertainty at each voxel. For this purpose, a full Bayesian approach would, however, be too costly. Instead, we compare, based on their correlation with the actual error, three simpler methods, namely, the dropout, the bootstrap and a modification of the U-Net. These methods can be easily adapted to other architectures. 200 patients with head and neck cancer were used in this work.
\end{abstract}

\section{Introduction}

To fight cancer with radiotherapy, a treatment plan must be devised such that it reaches the best tradeoff between disease control and adverse effects. The contours of the organs at risk (OAR) and the planning target volume (PTV) are drawn on a CT scan of the patient. Based on this information, the medical staff optimises the dose distribution to irradiate the PTV at the prescribed dose while respecting the dose constraints on the surrounding organs. However, this step is time consuming and involves many manual operations, which may lead to suboptimal and delayed treatments, compromising the patient outcome.

A major advance in treatment planning is the use of deep convolutional neural networks to predict the optimal dose distribution automatically and quickly. Recent literature shows good results with a U-Net architecture on different cancer locations $[1,2,3]$. These models predict the optimal 3D dose distribution from the patient's anatomy (OAR and PTV masks, as well as CT scan) in just a few seconds. However, in order to have a safe clinical implementation, physicians must know how certain the model is about the prediction. Adding a measure of uncertainty makes the prediction more interpretable and increases confidence in the prediction when the uncertainty is low. Areas of high uncertainty can be corrected manually by a specialist.

In this work, we first compare the prediction made with the U-Net by applying different dropouts and the bootstrap method. We then propose several

${ }^{*}$ M. H.-D. and A.M. B.-M. are funded by the Walloon region (PROTHERWAL/CHARP, grant 7289). J.A. L. is a Senior Research Associate with the F.R.S.-FNRS. 
methods to build a voxel-wise uncertainty map, indicating the degree of uncertainty in the model. The dropout and bootstrap methods are based on the voxel-wise standard deviation calculated on several dose estimates for the same patient. Finally, we modified the existing U-Net to predict the error of a trained model instead of the dose distribution itself. The methods require little or no architecture change and can be easily adapted to other models.

In the remainder of this paper, Section 2 presents our data and prediction model. Section 3 describes the implementation of the different abovementioned methods of uncertainty estimation. Next, Section 4 reports the results and discusses them. Section 5 concludes this work and sketches some perspectives.

\section{Patient data and dose prediction model}

A database of 200 patients (144 for training, 36 for validation, and for 20 testing) with head and neck cancer was used for this work.

A 3D U-Net architeture with dense connections [1][5] was used as dose prediction model, including 10 input channels for the anatomy of the patient ${ }^{1}$ and one output channel for the predicted dose distribution. The number of starting filters was 16 and learning rate was $3 \times 10^{-4}$. More information about the architecture can be found in [1].

\section{Methods of uncertainty estimation}

As full Bayesian modelling would be costly, we consider and compare three simpler approaches, namely, Monte Carlo dropout, bootstrap, and a second similar U-Net, with a different output, which predicts the uncertainty of the first U-Net.

Monte Carlo dropout is frequently used during neural network training to avoid overfitting and keep the model fairly general. In addition, by activating dropout during testing, we can obtain an approximation of the full Bayesian process and induce variation in the prediction of the dose distribution, which can later serve to compute the uncertainty [6]. We therefore trained 5 models with dropout rates ranging from 0.1 to 0.5 . We then evaluated $T=100$ times the dose distribution for each model and each of the 20 test patients, the predictions being all different due to the dropout activation during testing. For each voxel, we can compute the standard deviation on the $T$ predictions, which gives us an estimate of the uncertainty. In fact, a large standard deviation means that the model predicts different values for the same input and can be considered uncertain. The formulas that have been proposed in [7] are $\mathbb{E}\left(y^{*}\right) \approx \frac{1}{T} \sum_{t=1}^{T} \hat{y}^{*}\left(x, W_{t}\right)$, where $x$ is the network input, $W_{t}$ is the set of weights kept by dropout in testing and $\hat{y}^{*}$ is the model prediction. The set $W_{t}$ is drawn following a Bernouilli distribution on the whole set of network weights. Eventually, uncertainty $U\left(y^{*}\right)$ is approximated with $U\left(y^{*}\right) \approx \sqrt{\left(\frac{1}{T} \sum_{t=1}^{T} \hat{y}^{*}\left(x, W_{t}\right)^{2}\right)-\mathbb{E}\left(y^{*}\right)^{2}}$.

\footnotetext{
${ }^{1}$ target volume, CT scan, map indicating pixels where the dose can be non zero and each organ at risk (brainstem, spinal cord, right and left parotids, esophagus, larynx and mandible)
} 
Bootstrap relies on the same principle as MC dropout, except that the $T$ evaluations for each patient are obtained differently. For this method, we trained $T=20$ models with a dropout rate of 0.1 to avoid overfitting. For each model, the training set consists of 115 patients randomly selected among the 144 available. As soon as the 20 models were trained, we evaluated them on the 20 test patients. Variation in the predictions is no longer due to dropout but to the different models and actually to their different training sets. The same formulas as for MC dropout hold, with $W_{t}$ the set of weights of one model and $T=20$.

A secondary, slightly modified U-Net can also help predict uncertainty in a two-step fashion, by learning it from past examples.

First, a regular model with a dropout rate of 0.1 was trained ( model $_{\text {pred }}$ ) on the 144 test and 36 validation patients. The dose distribution of all 200 patients was evaluated with this model $T=50$ times, allowing us to compute the standard deviation and the mean absolute error the model commits when predicting the dose, i.e., the difference between the ground truth and the mean of the 50 predictions, for each patient.

Next, a U-Net with additional inputs and a modified output definition is trained to predict the error committed by model $_{\text {pred }}$. The dose distribution prediction and the standard deviation were added to the existing inputs (patient anatomical information). On the output side, the dose prediction was replaced by the actual error made by the model $_{\text {pred }}$. A new model ( model $_{\text {error }}$ ) was trained with the modified U-Net on the basis of the 144 training and 36 validation patients for whom the dose and standard deviation were previously calculated and was finally tested on the 20 test patients. The prediction of the error by the model $_{\text {error }}$ is considered as a measure of the uncertainty of the model $_{\text {pred }}$.

Eventually, dividing the uncertainty and error by the dose distribution in each voxel allows analysing the percentage of uncertainty and error relatively to the delivered dose in that voxel. This magnifies low uncertainties in OARs, for instance, where the dose is low but where small unexpected increments may cause serious side effects. A similar, approximate normalization is to divide the uncertainty and error by a predicted dose distribution as in practice the actual true dose would not be available yet.

\section{Results and discussion}

In this section, the performance of the different methods is compared, starting with the accuracy of the dropout and bootstrap methods in predicting the dose distribution. Then, the correlations obtained between the uncertainty maps and the actual errors are presented. Clinical dose assessment typically involves statistics, like means, medians, and percentiles of the dose distribution in some organs and volumes of interest, e.g., $D_{\max }$ or $D_{95}$ in PTV and $D_{\text {mean }}$ in OARs.

Figure 1 and Table 1 show the error made by the bootstrap method and the MC dropout on $D_{\text {mean }}$ and $D_{\max }$ for different OARs and PTVs. For OARs, the 
ESANN 2021 proceedings, European Symposium on Artificial Neural Networks, Computational Intelligence and Machine Learning. Online event, 6-8 October 2021, i6doc.com publ., ISBN 978287587082-7.

Available from http://www.i6doc.com/en/.

mean absolute error (MAE) on $D_{\text {mean }}$ is up to $0.63 \mathrm{~Gy}^{2}$ smaller with the bootstrap and 0.5Gy lower for $D_{2}$ (Brainstem). Regarding the PTV, the bootstrap is up to $0.18 \mathrm{~Gy}$ better than the dropout $\left(D_{95}\right)$. $D_{\max }$ is however better with the dropout for PTV56 and PTV63 (0.12 and 0.2Gy smaller), the bootstrap makes an error of $2.28 \%$ (PTV56) and $2.46 \%$ (PTV63) on the actual $D_{\max }$ value.
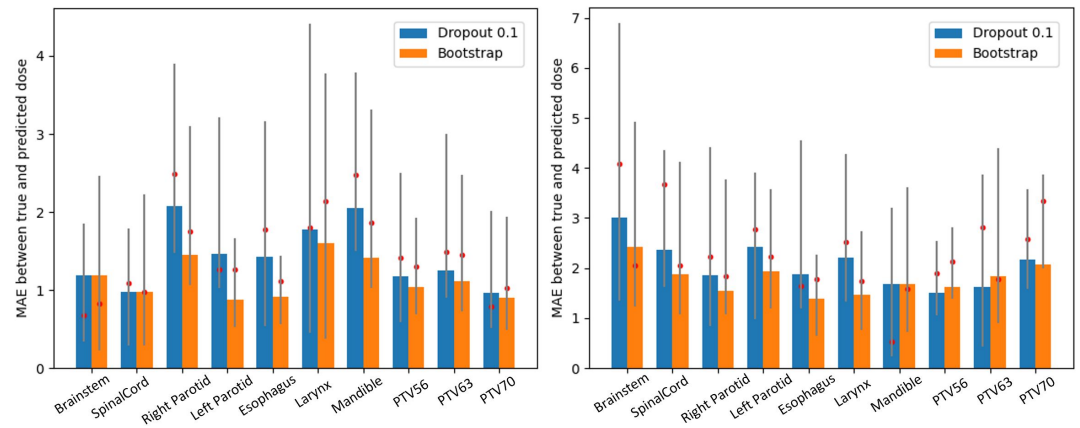

Fig. 1: On the left: Mean Absolute Error on $D_{\text {mean }}[\mathrm{Gy}]$. On the right : Mean Absolute Error on $D_{\max }[\mathrm{Gy}]$. The bars represent the mean on test patients, red point indicates the median and the grey line connects quantiles 0.25 and 0.75 .

\begin{tabular}{|c|c|c|c|c|c|c|c|}
\hline & PTV/OAR & Dropout=0.1 & Dropout=0.2 & Dropout=0.3 & Dropout=0.4 & Dropout=0.5 & Bootstrap \\
\hline Dmean & Brainstem & $1.19 \pm 1.64$ & $1.14 \pm 1.2$ & $1.28 \pm 1.58$ & $1.32 \pm 1.41$ & $1.21 \pm 1.45$ & $1.19 \pm 1.39$ \\
\hline & SpinalCord & $0.98 \pm 0.92$ & $1.13 \pm 0.91$ & $1.24 \pm 1.04$ & $1.13 \pm 0.99$ & $1.19 \pm 1.2$ & $0.98 \pm 0.98$ \\
\cline { 2 - 5 } & Right Parotid & $2.07 \pm 1.5$ & $2.08 \pm 1.26$ & $1.88 \pm 1.17$ & $1.35 \pm 1.01$ & $2.13 \pm 1.41$ & $1.46 \pm 0.93$ \\
\hline & Left Parotid & $1.46 \pm 1.21$ & $1.81 \pm 1.17$ & $1.51 \pm 1.16$ & $1.22 \pm 0.77$ & $1.26 \pm 0.89$ & $0.88 \pm 0.64$ \\
\hline & Esophagus & $1.42 \pm 1.16$ & $1.44 \pm 0.83$ & $0.93 \pm 0.66$ & $1.05 \pm 0.82$ & $0.89 \pm 0.84$ & $0.92 \pm 0.8$ \\
\hline & Larynx & $1.78 \pm 1.67$ & $2.04 \pm 1.9$ & $1.67 \pm 1.09$ & $1.84 \pm 1.98$ & $2.98 \pm 2.4$ & $1.6 \pm 1.36$ \\
\hline & Mandible & $2.05 \pm 1.37$ & $1.92 \pm 1.38$ & $1.61 \pm 0.97$ & $1.43 \pm 0.91$ & $2.31 \pm 1.95$ & $1.42 \pm 0.79$ \\
\hline D95 & PTV56 & $1.45 \pm 1.25$ & $1.52 \pm 1.39$ & $1.1 \pm 1.06$ & $1.54 \pm 0.99$ & $1.7 \pm 1.2$ & $1.39 \pm 1.05$ \\
\hline & PTV63 & $1.73 \pm 1.89$ & $1.98 \pm 1.86$ & $1.91 \pm 1.56$ & $1.69 \pm 1.92$ & $1.93 \pm 1.98$ & $1.63 \pm 1.87$ \\
\hline & PTV70 & $1.68 \pm 1.02$ & $1.75 \pm 1.46$ & $3.03 \pm 1.28$ & $1.37 \pm 1.0$ & $2.18 \pm 1.37$ & $1.5 \pm 0.93$ \\
\hline Dmax & PTV56 & $1.5 \pm 1.05$ & $1.73 \pm 1.34$ & $1.85 \pm 1.3$ & $1.69 \pm 1.08$ & $1.11 \pm 0.94$ & $1.62 \pm 0.93$ \\
\hline & PTV63 & $1.63 \pm 1.19$ & $2.17 \pm 1.35$ & $2.04 \pm 1.29$ & $2.27 \pm 1.19$ & $0.96 \pm 0.64$ & $1.83 \pm 1.42$ \\
\hline & PTV70 & $2.17 \pm 1.89$ & $2.55 \pm 1.53$ & $2.19 \pm 1.01$ & $2.69 \pm 1.27$ & $2.32 \pm 2.31$ & $2.08 \pm 0.96$ \\
\hline D2 & Brainstem & $2.99 \pm 2.48$ & $2.22 \pm 1.87$ & $2.52 \pm 2.17$ & $3.14 \pm 2.26$ & $2.44 \pm 2.09$ & $2.49 \pm 1.86$ \\
\hline & SpinalCord & $1.9 \pm 1.51$ & $1.85 \pm 1.28$ & $1.93 \pm 1.19$ & $2.01 \pm 1.38$ & $3.06 \pm 2.22$ & $1.82 \pm 1.43$ \\
\hline
\end{tabular}

Table 1: Comparison of mean error [Gy] and standard deviation [Gy] of the investigated methods for the different regions of interest.

The correlation between the uncertainties obtained and the error on the dose prediction was evaluated on the PTV and outside the PTV (Body-PTV). It is of maximum 0.47 (Body-PTV) and 0.1 (PTV) for the MC dropout, 0.513(BodyPTV) and 0.017(PTV) for the bootstrap, 0.5(Body-PTV) and 0.223(PTV) for the modified U-Net. The modified U-Net is therefore the method providing the

\footnotetext{
${ }^{2}$ The Gray (symbol Gy) is the SI unit of absorbed dose and is defined as the absorption of one joule of energy, in the form of ionizing radiation, per kilogram of matter, i.e. one gray $=1 \mathrm{~J} / \mathrm{kg}^{2}$ [8].
} 
ESANN 2021 proceedings, European Symposium on Artificial Neural Networks, Computational Intelligence and Machine Learning. Online event, 6-8 October 2021, i6doc.com publ., ISBN 978287587082-7. Available from http://www.i6doc.com/en/.

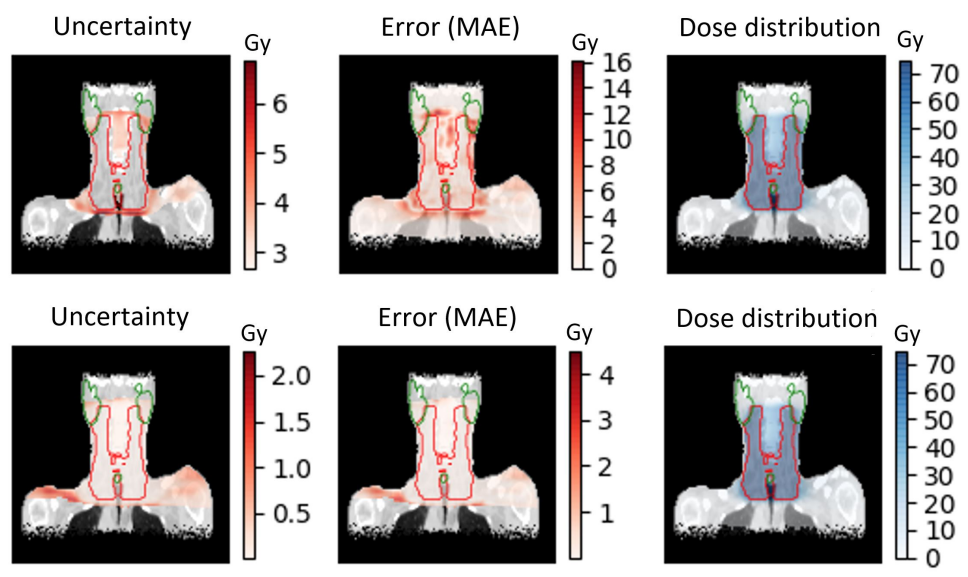

Fig. 2: Top : Comparison of the uncertainty obtained with the Modified UNet (left), the error on the dose distribution (middle) and the dose distribution (right). Bottom : Comparison of the uncertainty obtained with the Modified U-Net divided by the dose distribution (left), the error on the dose distribution divided by the dose distribution (middle) and the dose distribution (right)

best uncertainty map in general. A comparison of the uncertainty map with the error map is shown in Figure 2(top figure) for the modified U-Net. The selected slice is located at the centre of the tumor delineated in red on the picture. Right and left parotid, mandible and a part of the esophagus are represented in green.

When the uncertainty is divided by the dose distribution, the correlations are much better on the body-PTV: 0.7 (Body-PTV) and 0.215 (PTV) maximum for the MC dropout, 0.714 (Body-PTV) and 0.1 (PTV) for the bootstrap, 0.714 (Body-PTV) and 0.27 (PTV) for the modified U-Net, which is again the best indicator of the uncertainty. An illustration of this operation is shown in Figure 2(bottom) for the same patient and slice as used for the top Figure 2.

Finally, when we divide the uncertainty and error by the dose prediction rather than by the actual dose we find the following correlations: 0.55 (BodyPTV) and 0.17 (PTV) for the MC dropout, 0.56 (Body-PTV) and 0.09 (PTV) for the bootstrap, 0.56 (Body-PTV) and 0.274 (PTV) for the modified U-Net.

Concerning the prediction of the dose distribution, the MC dropout gives better overall results when the dropout rate is 0.1 . However, the bootstrap method offers better accuracy for most of the measured metrics, especially on the OARs for which the dose is lower than on the PTV. The only measured metric for which the bootstrap gives worse results is the $D_{\max }$, but with a relatively low error percentage. Regarding the prediction of the uncertainty measure, the U-Net model adapted to the prediction of the error provides the most correlated measure with the error on the PTV as well as one of the best correlations on the Body-PTV with similar results as the bootstrap. We notice a clear increase 
ESANN 2021 proceedings, European Symposium on Artificial Neural Networks, Computational Intelligence and Machine Learning. Online event, 6-8 October 2021, i6doc.com publ., ISBN 978287587082-7.

Available from http://www.i6doc.com/en/.

in the correlation when using dose distribution normalization. Although this result is theoretical as the exact dose distribution is unknown in a real case, it is encouraging and suggests the presence of an interesting relationship.

\section{Conclusion and perspectives}

We presented several methods that can give a relative measure of model uncertainty combined with a prediction of the dose distribution. These methods have the advantage of being cheap, unlike Bayesian modelling, as well as being easily adaptable to other architectures and even other domains of application. These affordable measures of uncertainty could be integrated into an active learning algorithm for which a database with a limited number of annotations would be available.The algorithm would identify regions of high uncertainty that would be reported to professionals who would be asked to manually annotate the images by optimising the dose distribution over a specific area by hand. The new annotations would subsequently extend the training data set. The areas that require annotation by a specialist are thus optimally and economically selected, avoiding the need to annotate the whole set of images. A similar idea of active learning was proposed in [9] where the error committed on the prediction by the model is predicted by an additional module and the unlabeled datasets with the largest predicted error were presented to a specialist to be labelled by hand.

\section{References}

[1] Nguyen D, Long T, Jia X, et al. A feasibility study for predicting optimal radiation therapy dose distributions of prostate cancer patients from patient anatomy using deep learning. Sci Rep. 2019;9(1):1076.

[2] Barragán-Montero AM, Nguyen D, Lu W, et al. Three-dimensional dose prediction for lung IMRT patients with deep neural networks: robust learning from heterogeneous beam configurations. Med Phys. 2019;46(8):3679-3691.

[3] Nguyen D, Jia X, Sher D, et al. 3D radiotherapy dose prediction on head and neck cancer patients with a hierarchically densely connected U-net deep learning architecture. Physics in Medicine \& Biology. 2019;64(6):065020.

[4] Babier A, Zhang B, Mahmood R, et al. OpenKBP: The open-access knowledge-based planning grand challenge. arXiv [physics.med-ph]. Published online November 28, 2020. http://arxiv.org/abs/2011.14076

[5] O. Ronneberger O, Fischer P, Brox T. U-Net: Convolutional Networks for Biomedical Image Segmentation. In: Medical Image Computing and Computer-Assisted Intervention - MICCAI 2015. Springer International Publishing; 2015:234-241

[6] Gal Y, Ghahramani Z. Dropout as a Bayesian Approximation: Representing Model Uncertainty in Deep Learning. In: International Conference on Machine Learning. PMLR; 2016:1050-1059

[7] Nguyen D, Sadeghnejad Barkousaraie A, Bohara G, et al. A comparison of Monte Carlo dropout and bootstrap aggregation on the performance and uncertainty estimation in radiation therapy dose prediction with deep learning neural networks. Phys Med Biol. 2021;66(5):054002.

[8] Murphy A, Dr J Bell D, et al. Gray (SI unit). https://radiopaedia.org/articles/gray-si-unit

[9] Yoo D, Kweon IS. Learning Loss for Active Learning. 2019 IEEE/CVF Conference on Computer Vision and Pattern Recognition (CVPR). Published online 2019. 